\title{
Diversidade de Resultados no Estudo do Transtorno de Déficit de Atenção e Hiperatividade
}

\author{
Célia G. Oliveira \\ Pedro B. Albuquerque ${ }^{1}$ \\ Universidade do Minho, Portugal
}

\begin{abstract}
RESUMO - Com este artigo pretende-se abordar a problemática da diversidade de dados na investigação do Transtorno de Déficit de Atenção e Hiperatividade (TDAH). Apresenta-se uma revisão da literatura centrada na heterogeneidade de conclusões relativas à caracterização do transtorno, à distinção dos subtipos, aos contextos de informação, às diferenças de gênero e à comorbidade. Na tentativa de compreender a disparidade de conclusões, salientam-se potenciais fatores explicativos, nomeadamente a heterogeneidade das amostras, a diversidade de metodologias e de procedimentos de investigação, entre outros. A revisão efetuada baseou-se, majoritariamente, em publicações referenciadas pelas bases de dados PsycInfo e ERIC, e, pontualmente, PubMed e Elsevier Direct.
\end{abstract}

Palavras-chave: TDAH; subtipos; crianças; diagnóstico.

\section{Results Diversity on the Study of Attention-Deficit Hyperactivity Disorder}

\begin{abstract}
With this paper we aimed at addressing the problem of data diversity in Attention-Deficit Hyperactivity Disorder (ADHD) research. We present a literature review based on the heterogeneity of findings about the characterization of the disorder, subtypes differentiation, sources of information, sex differences and comorbidities. In an effort to understand the variety of findings, we underline potential explanations, such as the sample's heterogeneity or the multiplicity of methods and procedures, among others. The review conducted was primarily based on PsycInfo and ERIC databases, but also, on PubMed and Elsevier Direct.
\end{abstract}

Keywords: ADHD; subtypes; children; diagnosis.

O Transtorno de Déficit de Atenção e Hiperatividade (TDAH) manifesta-se, essencialmente, por níveis clinicamente significativos de desatenção, hiperatividade e impulsividade, afetando frequentemente diversas áreas do funcionamento adaptativo, nomeadamente interpessoal, acadêmica ou familiar. A esse conjunto de características acresce a elevada incidência de transtornos comórbidos (American Psychiatric Association, 1994, 2000; Thapar, Langley, O'Donovan \& Owen, 2006). Dados recentes estimam uma prevalência mundial na ordem dos 5,2\%, maior no sexo masculino do que no feminino (Polanczyk, de Lima, Horta, Biederman \& Rohde, 2007). Além disso, a conquista progressiva de uma conceitualização desenvolvimental aponta para uma evolução com início precoce, passível de se prolongar pela idade adulta e de assumir um caráter crônico (Kessler \& cols., 2006). No entanto, apesar da elevada prevalência e das manifestações evidentes do transtorno, os resultados das investigações são frequentemente controversos e divergentes. Com a revisão aqui apresentada, pretende-se ilustrar a diversidade de dados produzidos em áreas essenciais do estudo do TDAH, como sejam a definição e caracterização do transtorno, a distinção dos tipos, os contextos de informação, as diferenças de gênero e a comorbidade. Nesse sentido, discutem-se alguns

1 Endereço para correspondência: Instituto de Educação e Psicologia, Universidade do Minho, Campus de Gualtar. 4710-057 Braga, Portugal. Tel. +351256604240 . Fax +351253604221 . E-mail: pedro.b.albuquerque@iep.uminho.pt. dos fatores que poderão contribuir para a heterogeneidade dos resultados e consequente dificuldade de sistematização de conclusões.

\section{(Re) Definições e Critérios de Diagnóstico}

Na origem do estudo sistematizado do transtorno encontra-se a formulação de Still (1902, citado por Barkley, 1997), o qual descreveu um grupo de crianças com características de agressividade, ausência de regras, desatenção, impulsividade e excessiva atividade, salientando as semelhanças comportamentais entre essas crianças e pacientes com lesão do lobo frontal. Sabe-se, atualmente, que essa região cerebral desempenha um papel primordial na inibição comportamental, atenção sustentada, autocontrole e planejamento do futuro (e.g., Barkley, 1997; Booth \& cols., 2005). Até os anos 60, prevaleceu uma concepção orgânica do transtorno, acompanhada de uma progressiva ênfase nas manifestações comportamentais. Por essa razão, o transtorno é incluído na segunda edição do Manual de Diagnóstico e Estatística dos Transtornos Mentais (DSM-II) com a designação de Reação Hipercinética da Infância (American Psychiatric Association, 1968). Durante a década de 70, os estudos destacaram o sintoma da hiperatividade sobre os sintomas restantes, mas no final dessa mesma década, a investigação passou a valorizar o sintoma de desatenção, dado o reconhecimento de que as crianças podiam ter déficits atencionais sem hiperatividade. 
Essa mudança motivou a redefinição e classificação do transtorno em dois tipos: Transtorno de Déficit de Atenção com Hiperatividade e Transtorno de Déficit de Atenção sem Hiperatividade (DSM-III, American Psychiatric Association, 1980).

No entanto, no decorrer dos anos 80 , vários estudos atribuíam à hiperatividade um papel fundamental e, por essa razão, na terceira edição revisada do DSM (DSM-III-R, American Psychiatric Association, 1987), o transtorno é redefinido como Transtorno de Déficit de Atenção com Hiperatividade e é eliminada a diferenciação em dois tipos. Essa mudança representou um recuo para uma classificação unidimensional e resultou, muito provavelmente, no aumento de falsos positivos (Manga, Fournier \& Navarredonda, 1999). O desenvolvimento da investigação acabou por sugerir uma correlação pouco significativa entre os fatores de atenção e da hiperatividade, mas muito significativa entre a impulsividade e atenção, por um lado, e a impulsividade e hiperatividade, por outro (Hinshaw, 1994). Em consonância, o DSM-IV (American Psychiatric Association, 1994, 2000) reintroduz a classificação multidimensional, agrupando, em tipos, os sintomas em que predomina a desatenção (tipo predominantemente desatento), a hiperatividade e a impulsividade (tipo predominantemente hiperativo-impulsivo) e considerando, ainda, um tipo combinado. Essa classificação procura harmonizar a complexidade subjacente à conceitualização do TDAH baseando-se nos critérios apontados pelos especialistas, admitindo a distinção introduzida pelo DSMIII e considerando os fatores de desatenção e hiperatividade sugeridos pela análise fatorial (Hinshaw, 1994; Manga \& cols., 1999).

Ainda no que respeita aos sistemas de classificação, importa considerar a descrição do grupo dos Transtornos Hipercinéticos proposta na décima edição do sistema de Classificação Internacional das Doenças (CID-10) (World Health Organization, 1996). Apesar do paralelo com a descrição do DSM-IV (American Psychiatric Association, 1994, 2000), a proposta do CID-10 apresenta diferenças relevantes, como seja o estabelecimento do diagnóstico dos Transtornos Hipercinéticos apenas na presença conjunta de sintomas de hiperatividade, desatenção e falta de persistência nas tarefas, excluindo os casos nos quais predominam apenas os sintomas de desatenção (tipo desatento do DSM-IV). Por outro lado, os sintomas de transtorno de conduta constituem a base para a classificação dos Transtornos Hipercinéticos em Transtorno de Conduta Hipercinética ou Transtorno Hipercinético, dependendo, respectivamente, da presença ou ausência de sintomas de transtorno de conduta associados à hiperatividade. Desse modo, contrariamente ao DSM-IV, não se admite a comorbidade entre TDAH e transtorno de conduta enquanto entidades nosológicas distintas. O diagnóstico de Transtorno Hipercinético é feito quando predominam os sintomas de desatenção e hiperatividade na ausência de problemas significativos de conduta. Esse diagnóstico assemelha-se ao do tipo combinado no DSM-IV (Sergeant, Geurts \& Oosterlaan, 2002; World Health Organization, 1996). Finalmente, para o diagnóstico diferencial, excluem-se todos os transtornos caracterizados por sintomas de desatenção ou de hiperatividade atribuídos aos transtornos de desenvolvimento, de ansiedade, de humor ou à esquizofrenia. $\mathrm{O}$ diagnóstico de comorbidade apenas é feito quando os sintomas de Transtorno Hipercinético revelam claramente a presença individual do mesmo (World Health Organization, 1996).

Pode-se concluir, então, que a conceptualização do TDAH não só tem sido alvo de sucessivas reformulações, como apresenta diferenças em função do sistema de classificação nosológica. Tais reformulações e divergências têm vindo a contribuir para a heterogeneidade das amostras e dos dados obtidos. Constituem exemplo disso, as variações de prevalência decorrentes do recurso a diferentes sistemas de diagnóstico (Skounti, Philalithis \& Galanakis, 2007; Taylor \& cols., 2004).

\section{Caracterização e Distinção de Tipos}

Os dados da investigação também variam em função dos tipos contemplados. Além disso, a distinção dos tipos entre si (American Psychiatric Association, 1994, 2000) requer aprofundamento, uma vez que as diferenças de sintomatologia predominante parecem refletir não só diferenças comportamentais, mas também cognitivas, etiológicas e desenvolvimentais, assim como necessidades terapêuticas distintas (Warner-Rogers, Taylor, Taylor \& Sandberg, 2000; Lane, 2004).

Em particular, o funcionamento cognitivo tem vindo a ser investigado como potencial fator de diferenciação dos tipos. No tipo desatento, a observação de maior incidência de déficits no processamento da informação tem levado alguns autores a questionar a sua inclusão no TDAH e a hipotetizar que se trata de um transtorno qualitativamente diferente, embora essa possibilidade careça ainda de sólida validação empírica (Carlson, Booth, Misung \& Canu, 2002; HuangPollock, Nigg \& Halperin, 2006).

A comparação do perfil neuropsicológico dos tipos foi objeto da meta-análise realizada por Lane (2004). As conclusões sugerem que o tipo combinado tende a mostrar melhores resultados em medidas de velocidade de processamento, atenção, QI de realização, memória e fluência; enquanto o tipo desatento tende a apresentar melhores resultados nas áreas de flexibilidade cognitiva, memória de trabalho, competência visuo-espacial, desempenho motor e linguagem. Na meta-análise de Frazier, Demaree e Youngstrom (2004), as crianças com TDAH apresentaram resultados globais de QI significativamente inferiores aos dos grupos de controle saudáveis, mas não foram encontradas diferenças entre os tipos. Contudo, os autores salientam a escassez de grupos desatentos incluídos nos estudos, o que poderia justificar os resultados, e apontam para a necessidade de aprofundamento desse aspecto. Efetivamente, um dos poucos estudos que contempla um grupo amplo de meninos com TDAH desatento revelou que este grupo apresentou um desempenho pior nos resultados globais de QI do que os tipos combinado e hiperativo/impulsivo (Warner-Rogers \& cols., 2000).

Finalmente, um dado importante na distinção dos tipos refere-se à caracterização dos respectivos déficits atencionais. Nesse âmbito, destaca-se o estudo de Collings (2003), realizado com uma amostra de meninos em idade escolar. Até então, a generalidade dos estudos não apontava diferenças entre os tipos nas medidas de atenção sustentada (Lane, 
2004). Contudo, Collings realizou uma avaliação detalhada do desempenho de cada um dos grupos numa tarefa de realização contínua, diferenciando três níveis de duração da tarefa e três intervalos inter-estímulos. Desse modo, pôde constatar que, por oposição ao tipo desatento, apenas o tipo combinado apresentava déficits de atenção sustentada revelados pelo acentuado declínio no desempenho da tarefa, e que o nível de vigilância do grupo desatento não se diferenciava significativamente do grupo de controle. Esses dados corroboram apenas parcialmente a hipótese proposta por Barkley (1997), segundo a qual o tipo desatento apresenta um déficit específico de vigilância, enquanto o tipo combinado se caracteriza essencialmente por déficits de inibição.

Mais recentemente, recorrendo ao mesmo tipo de tarefa, mas com intervalos inter-estímulos constantes, Huang-Pollock e cols. (2006) encontraram déficits de atenção sustentada em ambos os tipos, com o tipo combinado apresentando, adicionalmente, índices de maior ativação no estilo de resposta, coerentes com a característica comportamental de impulsividade. Isso sugere que o déficit de vigilância seria comum a ambos os tipos, diferenciando-os os níveis de ativação. Além disso, nenhum dos grupos apresentou déficits de atenção seletiva, à semelhança dos resultados apontados pela meta-análise de Bayens, Roeyers, e Walle (2006). Estes concluem que, apesar da profusa contradição de dados, as crianças com TDAH não parecem apresentar déficits significativos de atenção seletiva e que os déficits de atenção dividida são consistentes, mas não diferenciam os tipos entre si. Resultados semelhantes foram obtidos por Pasini, Paloscia, Alessadrelli, Porfirio e Curatolo, (2007) com um grupo de 50 meninos com TDAH, entre 8 e 14 anos de idade. Para além da atenção dividida, os grupos foram avaliados em um importante conjunto de tarefas executivas. Também nestas não foram encontradas diferenças entre os tipos, apenas entre o grupo TDAH e o grupo de controle em medidas de inibição e de memória de trabalho.

Com efeito, do ponto de vista cognitivo, o estudo das funções executivas tem assumido especial relevância, sobretudo pela semelhança encontrada entre as manifestações comportamentais do TDAH e os transtornos neuropsicológicos decorrentes de disfunções executivas, como as que resultam de lesões frontais (Barkley, 1997; Seidman, 2006). A meta-análise de Frazier e cols. (2004), que incluiu um conjunto vasto e diversificado de medidas neuropsicológicas, revelou, também, um pior desempenho dos grupos TDAH em medidas de natureza executiva por comparação com tarefas não-executivas. Os déficits executivos encontrados apóiam parcialmente a proposta de Barkley (1997) e referem-se à inibição comportamental, atenção e memória de trabalho. Contudo, os resultados para as áreas da auto-regulação afetiva, internalização do discurso, reconstituição e controle motor apresentaram menor consistência e necessidade de aprofundamento. No entanto, convém salientar que a hipótese de Barkley (1997) caracteriza essencialmente os tipos combinado e hiperativo-impulsivo, por considerar que o tipo desatento apresentaria características cognitivas únicas.

Apesar disso, conforme anteriormente se referiu, nem todos os estudos corroboram essa hipótese. Um exemplo disso são os resultados do estudo longitudinal de Hinshaw, Carte, Sami, Treuting e Zupan (2002) com meninas entre 6 e
12 anos. A comparação dos tipos desatento e combinado com um grupo de controle equivalente revelou o pior desempenho dos grupos TDAH em um conjunto alargado de tarefas executivas, mas os tipos não se diferenciaram entre si. Dados semelhantes foram obtidos por um criterioso estudo realizado apenas com meninos (Geurts, Verté, Oosterlaan, Royers \& Sergeant, 2005). Também numa amostra do sexo masculino, com 248 crianças entre 6 e 10 anos de idade, Gadow e cols. (2004) observaram um padrão de maior disfuncionalidade no tipo combinado, mas verificaram que a distinção comportamental e cognitiva dos tipos variava em função da fonte de informação considerada (apenas mães, apenas professores ou ambos) e que, contrariamente ao esperado, o tipo desatento não apresentava um resultado sistemático de pior desempenho acadêmico. Por tudo isso, é de salientar que a hipótese da disfunção executiva tem vindo a reunir progressiva validação para a caracterização do perfil cognitivo do TDAH, mas os dados obtidos não permitem diferenciar com segurança o perfil neuropsicológico dos tipos. Por outro lado, quando se utilizam grupos de comparação clínicos, constata-se que os déficits executivos não parecem ser exclusivos do TDAH (e.g., Brocki \& Bohlin, 2006; Geurts \& cols., 2005; Scheres \& cols., 2004; Willcutt, Pennington, Olson, Chabildas \& Hulslander, 2005).

Do ponto de vista comportamental, o TDAH combinado tem vindo a ser apontado como o mais comprometedor do funcionamento adaptativo, com maior incidência de problemas de relacionamento interpessoal e de comportamento exteriorizado, como sejam a agressividade, a impulsividade ou os transtornos opositivo e de conduta (e.g., Murphy, Barkley \& Bush, 2002; Short, Fairchild, Findling \& Manos, 2007). No que diz respeito aos comportamentos interiorizados, as diferenças entre os tipos tendem a extinguir-se quando se controlam aspectos fundamentais, como as fontes de informação, critérios de identificação, tamanho da amostra e respectivo poder estatístico para diferenciar grupos, bem como a presença ou ausência de comorbidade com transtornos exteriorizados (Bayens \& cols., 2006; Power, Costigan, Eiraldi \& Leff, 2004).

Uma outra linha de investigação é a que se refere à contribuição dos fatores familiares e genéticos para a distinção dos tipos. A título de exemplo, um estudo levado a cabo com uma amostra ampla de 386 pais e irmãos de crianças com TDAH revelou déficits no desempenho do Trailmaking Test apenas nos familiares das crianças do tipo combinado. Esse teste avalia a competência de controle atencional ao exigir que se alterne rapidamente entre estímulos de letras e números. No entanto, na generalidade das medidas executivas restantes não foram encontradas diferenças entre os familiares de cada um dos tipos (desatento versus combinado) (Nigg, Blaskey, Stawicki \& Sachek, 2004). Outros estudos comparam, geneticamente, gêmeos e irmãos de crianças com TDAH, a fim de determinar o efeito das influências ambientais e genéticas na manifestação dos tipos. Contudo, até ao momento, os resultados também não permitiram diferenciar, cabalmente, os tipos no plano genético. Essa é ainda uma linha de investigação em desenvolvimento (Thapar \& cols., 2006).

Em síntese, alguns estudos sugerem que as diferenças de natureza comportamental e cognitiva parecem demarcar o tipo desatento dos restantes tipos (sobretudo combinado), 
avançando a hipótese de essa demarcação refletir não só diferentes agrupamentos de sintomas, mas também déficits de natureza e etiologias distintas, com o tipo desatento constituindo-se como um transtorno qualitativamente diferente. Contudo, são ainda pouco consistentes os resultados que permitem confirmar tal hipótese (Bayens \& cols., 2006; Edwards \& cols., 2007; Gadow \& cols., 2004; Huang-Pollock $\&$ cols. 2006). Por outro lado, conforme fica claro a partir dos estudos analisados, os resultados na investigação dos tipos dependem, em boa medida, de aspectos essenciais como o controle de limitações metodológicas decorrentes da comorbidade, do contexto de identificação das amostras, dos grupos de comparação e do tipo de medidas utilizadas.

\section{Informantes e Contextos de Identificação}

Os estudos também divergem no contexto e fontes de identificação utilizados. Alguns privilegiam o contexto escolar, por considerarem que os professores estarão aptos para a identificação de comportamentos não-normativos, constituindo esta, frequentemente, a única fonte de informação (Brocki \& Bohlin, 2006; Epstein \& cols., 2005). Contudo, é possível obter resultados diferentes em função do contexto de identificação a que se recorre (Gadow \& cols., 2004;). O esclarecimento desse aspecto norteou o estudo longitudinal de Mannuzza, Klein e Moulton (2002) realizado durante 12 anos, com uma amostra de meninos entre 6 e 12 anos de idade. A amostra contemplou quatro grupos: meninos considerados hiperativos em casa e na escola (TDAH generalizado), meninos hiperativos na escola (assim considerados pelos professores, mas não pelos pais), meninos hiperativos no contexto familiar (assim considerados pelos pais, mas não pelos professores) e um grupo de controle constituído por meninos sem transtorno. Do seguimento dos participantes, foi possível distinguir dois grandes grupos: o dos participantes com TDAH generalizado e com TDAH em contexto escolar, por um lado, e o dos participantes com TDAH em contexto familiar controle e sem TDAH, por outro lado, tendo os primeiros apresentado uma maior prevalência de transtorno anti-social, problemas comportamentais mais severos, bem como maiores níveis de abandono e de insucesso escolar. Nesse estudo, a identificação em contexto escolar parece ser mais fidedigna e caracterizar um grupo com maior severidade comparativamente à identificação em contexto familiar.

Pode-se sempre levantar questões quanto à adequação das amostras baseadas numa única fonte e contexto de informação, dada a impossibilidade de determinar o impacto da disfunção em diferentes contextos de vida e, ainda, a impossibilidade de controlar o risco de uma avaliação menos precisa na ausência de fontes de comparação. Dados recentes sugerem que variáveis ambientais, como o tamanho das turmas e a proveniência cultural dos sujeitos, podem influenciar a percepção dos professores na identificação do TDAH e contribuir para uma excessiva referenciação dos alunos (Havey, Olson, McCormick \& Cates, 2007). Por outro lado, as investigações que contemplam apenas as informações de um dos pais (normalmente, as mães) parecem correr ainda maiores riscos de inexatidão, apresentando maior inconsis- tência do que os relatos dos professores que detêm, inclusive, uma maior associação com os resultados neurocognitivos (Thapar \& cols., 2006). Ainda no que aos informantes se refere, são igualmente conhecidas as limitações inerentes aos auto-relatos das crianças e que decorrem geralmente da falta de maturidade necessária para compreender e reportar com precisão estados emocionais, ou mesmo, a intensidade e duração dos sintomas. No caso dos transtornos exteriorizados de comportamento isso parece especialmente válido, sobretudo em idades inferiores a 10 anos (Achenbach, 1987, citado por Burt, Krueger, McGue \& Iacono, 2001).

Em resumo, não se trata de desvalorizar qualquer fonte, mas de reforçar a importância de se recorrer a várias simultaneamente. $\mathrm{O}$ que parece verificar-se é que a consideração de cada uma das fontes, isoladamente, aumenta a probabilidade de inexatidão na identificação do TDAH, até porque são habitualmente baixos os valores de concordância entre informantes (Thapar \& cols., 2006). Além disso, a identificação dos participantes a partir de um único contexto contraria o estipulado pelo DSM-IV (American Psychiatric Association, 1994, 2000), no qual apenas se admite o diagnóstico do transtorno quando os prejuízos decorrentes do quadro sintomático atingem dois ou mais contextos de vida.

\section{Diferenças de Gênero}

Um outro problema no estudo do TDAH refere-se à caracterização do transtorno no sexo feminino. As dificuldades decorrem, aparentemente, da menor prevalência nesse gênero e, consequentemente, menor representatividade das amostras que, muito frequentemente, são constituídas apenas por meninos (Seidman \& cols., 2005). Recentemente, Staller e Faraone (2006) defenderam que o sexo feminino estaria subdiagnosticado devido à relevância atribuída aos sintomas de hiperatividade e impulsividade. Efetivamente, do ponto de vista comportamental, as meninas parecem apresentar menor prevalência de comportamentos exteriorizados e, em particular, de transtorno de conduta comórbido. Alguns dados apontam uma probabilidade 2,2 vezes maior de ocorrência do tipo desatento no sexo feminino (Biederman \& cols., 2002, citado por Staller \& Faraone, 2006). Por outro lado, a predominância de sintomas de desatenção contribui para uma detecção mais tardia do problema, frequentemente após 7 anos de idade. No seu conjunto, esses fatores poderiam contribuir para uma representação diminuída do sexo feminino e, consequentemente, deflacionar os valores de prevalência.

Apesar da plausibilidade dessa hipótese, nem todos os estudos confirmam a maior incidência do tipo desatento nas meninas. Neuman e cols. (2005) analisaram uma amostra de 564 famílias com pares de gêmeos entre 7 e 18 anos, nos quais havia sido diagnosticado TDAH em pelo menos um deles. $\mathrm{O}$ estudo incluiu também um grupo de controle com 183 famílias. Baseados nos critérios do DSM-IV, os resultados apontaram uma maior prevalência do tipo desatento nos meninos e do tipo combinado nas meninas, embora os meninos apresentassem maior severidade na generalidade dos sintomas. Os autores concluíram que o tipo desatento poderia estar sub-identificado e, consequentemente, sub- 
intervencionado nos dois sexos, uma vez que a maioria das amostras é composta por meninos do tipo combinado.

Precisamente com o objetivo de colmatar a escassez de investigação no sexo feminino, alguns estudos são realizados exclusivamente com meninas, ou, em alternativa, com amostras representativas do sexo feminino. Isso foi verificado no estudo de Rumsey (2004), o qual comparou 137 meninos e 65 meninas, entre 6 e 16 anos, em 13 medidas do funcionamento executivo. A única comorbidade admitida foi a dislexia, cuja presença serviu de critério para a constituição dos grupos. No grupo sem comorbidade, ambos os sexos apresentaram um pior desempenho na generalidade das tarefas comparativamente ao grupo de controle não-clínico. No entanto, os sexos não se diferenciaram entre si no desempenho global. Por sua vez, o grupo com TDAH e dislexia destacou-se, sobretudo, pela maior incidência de déficits na memória de trabalho verbal, mas, uma vez mais, não se encontraram diferenças significativas entre meninos e meninas. Ao que tudo indica, os déficits de memória de trabalho verbal constituem uma característica distintiva daquele tipo de comorbidade, mas não do gênero. Com efeito, do ponto de vista neuropsicológico, os déficits executivos nas meninas em idade escolar parecem ser análogos aos apresentados pelos meninos e, à semelhança do que já se referiu na caracterização dos tipos, também não são claras as diferenças de funcionamento executivo quando se contrastam os tipos combinado e desatento no sexo feminino (Hinshaw \& cols., 2002). Resultados idênticos têm sido corroborados por estudos transversais e por estudos longitudinais que analisam o desempenho cognitivo dos sexos na infância e na adolescência ou no início da idade adulta (Fischer, Barkley, Smallish, \& Fletcher, 2005; Hinshaw, Carte, Fan, Jassy \& Owens, 2007; Rucklidge \& Tannock, 2002; Seidman \& cols., 2005).

Do ponto de vista comportamental, o tipo combinado parece constituir um fator de risco para a ocorrência de maus-tratos no sexo feminino. Tais conclusões derivam da comparação de uma amostra de 140 meninas com TDAH, em idade escolar (6-12 anos), com um grupo cultural e sócioeconomicamente equivalente $(\mathrm{N}=88)$. Para além da maior incidência de maus-tratos no grupo TDAH, foi igualmente observada maior frequência de comportamentos de agressividade e maior rejeição social, decorrendo desse estudo importantes questões relativas ao papel dos maus tratos na etiologia e/ou agravamento do transtorno (Briscoe-Smith \& Hinshaw, 2006). Em conformidade com revisões anteriores, na meta-análise levada a cabo por Gershon (2002), as meninas tendem a apresentar maior prevalência de problemas interiorizados, em detrimento de problemas exteriorizados. Contudo, uma vez mais, se constata a necessidade de contextualização dos dados. De fato, nas amostras clínicas, os meninos tendem a apresentar índices mais elevados de problemas de comportamento, contudo, essas diferenças não são consistentes quando se recorre a amostras não-clínicas (obtidas no contexto escolar ou na população em geral). Nestas, poderá observar-se algum efeito da fonte de informação utilizada, com os pais a apontarem índices idênticos de problemas de comportamento em ambos os sexos, e com os professores, em observações no contexto escolar, a indicarem uma maior severidade daquele tipo de problemas nos meninos (Derks,
Hudziak \& Boomsma, 2007). Outros estudos, porém, não encontraram diferenças significativas entre os sexos quando compararam contextos ou fontes de informação (e.g., DuPaul \& cols., 2006; Graetz, Sawyer, Baghurst \& Ettridge, 2006). Para além das abordagens cognitivas e comportamentais, poderiam ser mencionadas outras, como é o caso dos estudos genéticos, ou de imagiologia cerebral, cujos contributos para o conhecimento do transtorno no sexo feminino se encontram também em desenvolvimento e poderão ajudar a clarificar as semelhanças e diferenças entre os sexos (e.g., Derks \& cols., 2007; Goos, Ezzatian \& Schachar, 2007; Hermens, Kohn, Clarke, Gordon \& Williams, 2005).

De um modo geral, os dados das investigações aqui apresentadas indicam a necessidade de aprofundamento das especificidades de cada um dos gêneros. $\mathrm{O}$ esclarecimento dos dados aparece associado à necessidade de controlar questões metodológicas importantes, como a possibilidade de uma menor incidência de comportamentos exteriorizados nas meninas, a maior probabilidade de viés na identificação das mesmas por pais e professores, o recurso predominante a amostras clínicas masculinas e do tipo combinado ou o aprofundamento da comparação de cada um dos sexos com grupos comórbidos e com grupos clínicos (Derks \& cols., 2007; Nøvik \& cols., 2006; Seidman \& cols., 2005; Staller \& Faraone, 2006).

\section{Comorbidade}

A elevada prevalência de transtornos comórbidos constitui uma dificuldade acrescida no estudo do TDAH, sobretudo porque os dados sugerem que a comorbidade constitui a regra e não a exceção. Para além do impacto sobre as manifestações do transtorno, a comorbidade coloca dificuldades na constituição dos grupos, uma vez que se exige um rigoroso, frequentemente difícil, diagnóstico diferencial. Na revisão levada a cabo por Biederman (2005), os valores estimados de comorbidade nas crianças com TDAH são superiores a $60 \%$ para o transtorno desafiador opositivo (TDO), a 15\% para o transtorno de conduta (TC), a $25 \%$ para os transtornos de humor e de aprendizado, e aproximam-se dos $30 \%$ para os transtornos de ansiedade. Igualmente elucidativos são os dados resultantes do Multi-Modal Treatment Study (MTA), um importante estudo promovido pelo National Institute of Mental Health nos Estados Unidos da América, do qual resultou a avaliação e seguimento de 579 crianças com TDAH combinado, entre 7 e 9,9 anos de idade (MTA Cooperative Group, 1999). De acordo com Epstein e cols. (2007), essa é considerada uma das amostras mais bem definidas e estudadas no âmbito do TDAH. Os dados obtidos revelaram que $40 \%$ das crianças apresentavam comorbidade com transtorno desafiador opositivo e $14 \%$ com transtorno de conduta, diagnosticados segundo o DSM-IV. Além disso, 34\% da amostra apresentou comorbidade com algum tipo de transtorno de ansiedade à luz do DSM-III-R (MTA Cooperative Group, 1999). Para esses últimos, revisões mais recentes apontam valores semelhantes de comorbidade (Biederman, 2005; Schatz \& Rostain, 2006). 
A elevada comorbidade no TDAH foi igualmente corroborada em um estudo realizado no Brasil, com uma amostra de 36 participantes entre 6 e 16 anos de idade. A análise dos índices de comorbidade revelou uma prevalência na ordem dos $86 \%$, dos quais $57 \%$ se referiam à co-ocorrência de pelo menos dois transtornos a par do TDAH (Souza, Serra, Mattos \& Franco, 2001). Índices significativos de comorbidade foram, igualmente, apresentados por Barkley (1999, citado por Decker, McIntosh, Kelly, Nicholls \& Dean, 2001) ao indicar que $44 \%$ dos sujeitos com hiperatividade apresentaram um transtorno comórbido, 32\% apresentaram dois e 11\% apresentaram pelo menos três. Todos esses dados salientam um outro aspecto determinante e que se refere à possibilidade da comorbidade não se limitar a um único transtorno, podendo abranger dois ou mais transtornos adicionais. De entre os transtornos mais frequentes destacam-se os transtornos desafiador opositivo e de conduta, os transtornos do humor e da ansiedade e, ainda, os transtornos de aprendizado (Biederman, 2005; Kadesjö \& Gillberg, 2001).

A investigação dos efeitos da comorbidade sobre as manifestações do TDAH levou alguns autores a sugerirem a classificação do transtorno com base no tipo de comorbidade presente, decorrendo daqui um novo desafio para a investigação (e.g., Faraone, Biederman \& Monuteaux, 2001; Gau, 2001). Alguns estudos sugerem que a comorbidade TDAH/TC configura um tipo clinicamente severo, provavelmente assente na partilha de fatores genéticos (Nadder, Rutter, Silberg, Maes \& Eaves, 2002; Thapar, Harrington \& McGuffin, 2001). Assim sendo, o estudo da comorbidade poderá ajudar a clarificar aspectos relativos às manifestações, etiologia e evolução do transtorno. A esse propósito, alguns resultados indicam que comorbidades distintas resultam em prognósticos significativamente diferentes, como é o caso, por exemplo, da comorbidade entre TDAH e transtornos de aprendizado, que parece constituir um fator de risco acrescido para um percurso acadêmico comprometido (Faraone \& cols., 2002), em contraste com a presença de problemas de conduta que prognosticam o risco de desenvolvimento de comportamentos de agressividade e delinquência (Burt \& cols., 2001).

De modo semelhante, o estudo longitudinal de Gau (2001) revelou que, na infância, a comorbidade TDAH/TDO contribui para o posterior desenvolvimento de depressão, enquanto a comorbidade precoce com depressão ou transtorno de conduta promove o risco de abuso ou dependência de substâncias na juventude. Dados semelhantes para a comorbidade TDAH/ TDO foram recentemente observados com um estudo desenvolvimental que comparou crianças com e sem comorbidade $(\mathrm{N}=246)$. A presença de transtorno desafiador opositivo apareceu associada a índices mais elevados de depressão em faixas etárias mais avançadas ( 9 - 11,7 anos), por comparação com idades anteriores (6,6 - 9 anos) onde a presença de TDO não foi determinante dos índices de depressão (Ostrander, Crystal \& August, 2006). Também, recentemente, Harpold e cols. (2007) constataram que adultos TDAH com história de transtorno desafiador opositivo apresentavam maior disfuncionamento psicosssocial e índices mais elevados de comorbidade psiquiátrica, comparativamente a um grupo sem história de TDO comórbido.
Para além dos efeitos da comorbidade sobre a evolução e manifestações comportamentais do transtorno, são igualmente investigados os efeitos sobre o perfil cognitivo. Geurts, Verté, Oosterlaan, Royers e Sergeant (2004) avaliaram meninos entre 6 e 12 anos de idade em cinco domínios centrais do funcionamento executivo, nomeadamente: inibição, memória de trabalho visual, planeamento, flexibilidade cognitiva e fluência verbal. Os grupos TDAH, com $(\mathrm{N}=34)$ e sem $(\mathrm{N}=20)$ transtornos do comportamento comórbidos (transtorno de conduta e/ou desafiador opositivo), foram constituídos com recurso a vários métodos e ao critério de concordância inter-informantes (pais e professores). Por comparação com um grupo de controle não-clínico, os dois grupos clínicos revelaram um comprometimento significativo nas áreas de inibição e fluência verbal. Contudo, não se distinguiram entre si, sugerindo a ausência de diferenças claras entre o perfil executivo dos meninos TDAH com e sem transtornos do comportamento comórbidos. No sentido oposto, algumas investigações sugerem que a presença de transtornos do comportamento agrava os déficits de inibição comportamental e que é, por isso, determinante de um estilo de resposta impulsivo (Van der Meere \& cols., 2005, citado por Raymaekers, Antrop, Van der Meere, Wiersema \& Roeyers, 2007).

Um dado relativamente consistente parece ser o da maior severidade dos déficits de memória de trabalho verbal na comorbidade com os transtornos de leitura. Esses resultados adquirem robustez ao verificar-se sua ocorrência em diferentes faixas etárias e em ambos os sexos (Rucklidge \& Tannock, 2002; Rumsey, 2004). No entanto, apesar dessa particularidade, os déficits de memória de trabalho não são exclusivos desse tipo de comorbidade, podendo observar-se, isoladamente, nos grupos com TDAH ou com transtornos de aprendizado (Martinussen \& Tannock, 2006; Willcutt \& cols., 2005). Também noutros domínios não são claras as diferenças no perfil cognitivo desses grupos. Willcutt e cols., por exemplo, encontraram déficits executivos comuns a um grupo com transtornos de leitura e a outro com TDAH. Esses grupos não se distinguiram em tarefas de inibição comportamental, velocidade de processamento e flexibilidade cognitiva. Além disso, o grupo comórbido apenas se distinguia por apresentar um perfil cognitivo correspondente à combinação dos déficits apresentados por cada um dos grupos puros. Nesse sentido, a diferenciação do perfil executivo do TDAH e dos transtornos de leitura também requer aprofundamento.

A par dessas questões, a elevada prevalência desse tipo de comorbidade tem gerado grande debate em torno da (in) dissociação desses transtornos. Algumas linhas de investigação defendem que se trata de problemas singulares, mas frequentemente associados, por oposição aos que concebem essa comorbidade como uma única e distinta entidade nosológica, com base em estudos dedicados à investigação de uma etiologia comum a ambos os transtornos (Barkley, 2006; Willcutt \& cols., 2005; Willcutt, Pennington, Olson \& DeFries, 2007). São igualmente inconclusivos os dados que comparam o funcionamento executivo no TDAH e nos Transtornos do Espectro do Autismo. Diversos estudos constatam a existência de déficits executivos em ambos os tipos de transtorno, mas não são claras, nem consistentes, as 
diferenças entre o perfil executivo dos grupos, em particular no que respeita às competências de inibição comportamental. É possível que parte da inconsistência dos resultados se deva, precisamente, à ausência de controle da comorbidade, o que se verifica em alguns dos estudos que comparam estes transtornos (Corbett \& Constantine, 2006; Raymaekers \& cols., 2007). Outros tipos de comorbidade poderiam, ainda, ser referidos, como são o caso da epilepsia ou dos transtornos de tiques ou de Tourette. Particularmente, nesses casos, para além das questões comuns ao estudo da comorbidade no TDAH, como sejam os efeitos observados, a evolução ou a etiologia, colocam-se ainda questões prementes para a intervenção farmacológica, nomeadamente no que respeita à interação entre os fármacos utilizados para tratar cada um dos transtornos individualmente (e.g., Gonzalez-Heydrich $\&$ cols., 2007; Robertson, 2006).

Finalmente, considerando a elevada prevalência de transtornos comórbidos, a investigação contrasta, ainda, os efeitos de diferentes tipos de comorbidade. Isso mesmo foi observado a partir de uma amostra $(\mathrm{N}=499)$ retirada do estudo MTA (Newcorn \& cols., 2001) Dois tipos de comorbidade foram contrastados: TDAH/ Transtornos do Comportamento (TC ou TDO), por um lado, e TDAH/ Transtornos da Ansiedade, por outro. Da avaliação constou a administração de uma tarefa de realização contínua. Nesta, todos os grupos apresentaram elevados índices de desatenção e impulsividade. Contudo, as crianças TDAH/Transtornos de Comportamento revelaram valores mais elevados de impulsividade do que de desatenção, observando-se o padrão oposto no grupo com Transtornos de Ansiedade. No mesmo sentido, a já mencionada revisão de Schatz e Rostain (2006) sugere que a presença de transtornos de ansiedade no TDAH parece diminuir, parcialmente, a impulsividade e os déficits de inibição. Por outro lado, a diminuição nos déficits de inibição aparece, simultaneamente, associada a um pior desempenho em medidas atencionais. Entretanto, não é ainda claro o papel desempenhado pela ansiedade, uma vez que se desconhece se a mesma constitui um mecanismo alternativo de controle da impulsividade, ou se representa apenas uma consequência dos sintomas de TDAH. Ainda na investigação dos efeitos da comorbidade, destacam-se os transtornos afetivos, nomeadamente os transtornos depressivos e o transtorno bipolar. A compreensão dos mecanismos subjacentes a esse tipo de comorbidade tem apontado o papel relevante de variáveis como o desempenho acadêmico, a competência social ou a presença de transtornos do comportamento, mas essa é também uma linha de investigação em desenvolvimento (Herman, Lambert, Ialongo \& Ostrander, 2007; Ostrander \& cols., 2006).

Em síntese, a grande diversidade e prevalência de transtornos comórbidos constitui um tópico de estudo premente para a compreensão da natureza e heterogeneidade de perfis do TDAH, bem como para o aperfeiçoamento do diagnóstico diferencial. Com efeito, partindo dos dados da investigação, fica claro que não é possível ignorar o impacto da comorbidade, quer na avaliação clínica, quer na constituição das amostras de investigação, o que nem sempre se verifica, podendo contribuir para a diversidade e inconsistência dos resultados (Raymaekers \& cols., 2007; Schatz \& Rostain, 2006).

\section{Comentários Finais}

$\mathrm{O}$ avanço da investigação do TDAH ainda requer o aprofundamento de áreas como a etiologia do transtorno e das respectivas comorbidades, o estabelecimento de critérios de diagnóstico desenvolvimentalmente adequados, o aprofundamento das especificidades relativas aos tipos e ao gênero, a avaliação dos fatores de risco e de proteção ou, ainda, a identificação de endofenotipos exclusivos do TDAH (e.g., Brocki \& Bohlin, 2006; Derks \& cols., 2007; Epstein \& cols., 2007; Spencer, Biederman \& Mick, 2007). Com este artigo, pretendeu-se alertar para algumas das dificuldades que se colocam no estudo do transtorno e, simultaneamente, promover uma análise mais objetiva dos inúmeros dados que se publicam na área. Conforme se conclui da revisão da literatura, é possível que pelo menos uma parte da inconsistência dos resultados derive da dificuldade de controle das inúmeras questões metodológicas que os afetam, nomeadamente os critérios de constituição das amostras, as fontes e contextos de informação, os instrumentos de avaliação utilizados, a faixa etária e gênero sexual em estudo, a classificação em tipos, o despiste de comorbidades, a comparação com grupos clínicos ou saudáveis, entre outros. A consideração, ou não, desses e outros aspectos, é frequentemente suficiente para produzir resultados diversos.

Por tudo isto, interrogamo-nos se será o TDAH tão amplamente heterogêneo ou se, em vez disso, o atual conhecimento do transtorno reflete, sobretudo, a heterogeneidade da investigação. Em nosso entender, à semelhança das tentativas de consenso acerca da conceitualização do transtorno (e.g., Barkley \& cols., 2002; Kutcher \& cols., 2004; NIH, 2000; Taylor \& cols., 2004), seria crucial a discussão de linhas orientadoras do estudo na área, com o objetivo de promover o desenvolvimento de critérios e metodologias que contribuíssem para uma mais clara e rigorosa comparação da extensa investigação produzida e, consequentemente, para o paulatino esclarecimento das múltiplas polêmicas que a caracterizam.

\section{Referências}

American Psychiatric Association (1968). Diagnostic and statistical manual of mental disorders ( $2^{\text {nd }}$ ed.). Washington, DC: American Psychiatric Association.

American Psychiatric Association (1980). DSM-III, Diagnostic and statistical manual of mental disorders ( $3^{\text {rd }}$ ed.). Washington, DC: American Psychiatric Association.

American Psychiatric Association (1987). DSM-III-R, Diagnostic and statistical manual of mental disorders ( $3^{\text {rd }}$ ed., reviewed). Washington, DC: American Psychiatric Association.

American Psychiatric Association (1994). DSM-IV, Manual de diagnóstico e estatística das perturbações mentais ( $\left.4^{\mathrm{a}} \mathrm{ed}.\right)$. Lisboa: Climepsi.

American Psychiatric Association (2000). DSM-IV-R, Manual de diagnóstico e estatística das perturbações mentais ( $4^{\mathrm{a}} \mathrm{ed}$. revisada). Lisboa: Climepsi.

Barkley, R. A. (1997). ADHD and the nature of self-control. New York: Guilford Press. 
Barkley R. A., Cook, E. H., Dulcan, M., Campbell, S., Prior, M., Atkins, M., Gillberg, C., Solanto-Gardner, M., Halperin, J., Bauermeister, J. J., Pliszka, S. R., Stein, M. A., Werry, J. S., Sergeant, J., Brown, R. T., Zametkin, A., Anastopoulos, A. D., McGough, J. J., DuPaul, G. J., Faraone, S. V., Levy, F., Fischer, M., Biederman, J., Hartung, C., Houghton, S., Carlson, G., Johnston, C., Spencer, T. J., Tannock, R., Diamond, A., Whalen, C., Hinshaw, S. P., Quay, H., Piacentini, J., Firestone, P., Mannuzza, S., Abikoff, H., McBurnett, K., Pfiffner, L., Bukstein, O., Winters, K. C., DeKlyen, M., Hechtman, L., Carlson, C., Lynam, D. R. ; Tolan, P. H., Loney, J., Koplewicz, H. S., Milich, R., Greenhill, L., Mash, E. J., Schachar, R., Taylor, E., Hoza, B., Rapport, M. D., Pennington, B., Thapar, A., Teeter, A., Shapiro, S., Sadeh, A., Leventhal, B. L., Bird, H. R., Paternite, C. E., Fristad, M. A., Molina, B., Eyberg, S., McGee, R., Shelton, T. L., Evans, S. W., Loo, S. K., Pelham, W., Hodgens, J. B., Sagvolden, T., Brown, T. E., Connor, D. F., Waschbusch, D. A., Murphy, K. R., Aman, M., Corbett, B., Anderson, D. L., Weyandt, L. L., Gordon, M., \& Lewandowski, L. (2002) Consensus statement on ADHD. European Child \& Adolescent Psychiatry 11, 96-98.

Barkley, R. A. (2006). Comorbidity of adult ADHD with learning and executive function disorders. Primary Psychiatry, 13 (3, Suppl.), 1-16.

Bayens, D., Roeyers, H., \& Walle, J. V. (2006). Subtypes of attention-deficit hyperactivity disorder (ADHD): Distinct or related disorders across measurement levels? Child Psychiatry and Human Development, 36, 403-417.

Biederman, J. (2005). Attention-deficit hyperactivity disorder: A selective overview. Biological Psychiatry, 57, 1215-1220.

Booth, J. R., Burman, D. D., Meyer, J. R., Lei, Z., Trommer, B. L., Davenport, N. D., Li, W., Parrish, T. B., Gitelman, D. R., \& Mesulan, M. M. (2005). Larger deficits in brain networks for response inhibition than for visual selective attention in attention deficit hyperactivity disorder (ADHD). Journal of Child Psychology \& Psychiatry, 46, 94-111.

Briscoe-Smith, A. M., \& Hinshaw, S. P. (2006). Linkages between child abuse and attention-deficit hyperactivity disorder in girls: Behavioral and social correlates. Child Abuse \& Neglect, 30, 1239-1255.

Brocki, K. C., \& Bohlin, G. (2006). Developmental change in the relation between executive functions and symptoms of $\mathrm{ADHD}$ and co-occurring behaviour problems. Infant and Child Development, 15, 19-40.

Burt, S. A., Krueger, R. F., McGue, M., \& Iacono, W. G. (2001). Sources of covariation among attention deficit hiperactivity disorder, oppositional defiant disorder, and conduct disorder: The importance of shared environment. Journal of Abnormal Child Psychology, 110, 516-525.

Carlson, C. L., Booth, J. E., Shin, M., \& Canu, W. H. (2002). Parent-, teacher-, and self-rated motivational styles in ADHD subtypes. Journal of Learning Disabilities, 35, 104-113.

Collings, R. D. (2003). Differences between ADHD inattentive and combined types on the CPT. Journal of Psychopathology and Behavioral Assessment, 25, 177-189.

Corbett, B. A., \& Constantine, L. J. (2006). Autism and attention deficit hyperactivity disorder: Assessing attention and response control with the integrated visual and auditory continuous performance test. Child Neuropsychology, 12, 335-348.
Decker, S. L., McIntosh, D. E., Kelly, A. M., Nicholls, S. K., \& Dean, R. S. (2001). Comorbidity among individuals classified with attention disorders. International Journal on Neuroscience, 110, 43-54.

Derks, E. M., Hudziak J. J., \& Boomsma D. I. (2007). Why more boys than girls with ADHD receive treatment: A study of dutch twins. Twin Research and Human Genetics, 10, 765-770.

DuPaul, G. J., Jitendra, A. K., Tresco, K. E., Vile Junod, R. E., Volpe, R. J., \& Lutz, J. G. (2006). Children with attention deficit hyperactivity disorder: Are there gender differences in school functioning? School Psychology Review, 35, 292-308.

Edwards, M. C., Gardner, E. S., Chelonis, J. J., Schulz, E. G., Flake, R. A., \& Diaz, P. F. (2007). Estimates of the validity and utility of the Conners' Continuous Performance Test in the assessment of inattentive and/or hyperactive-impulsive behaviors in children. Journal of Abnormal Child Psychology, 35, 393-404.

Epstein, J. N., Willoughby, M., Valencia, E. Y., Tonev, S. T., Abikoff, H. B., Arnold, L. E., \& Hinshaw, S. P. (2005). The role of children's ethnicity in the relationship between teacher ratings of attention-deficit hyperactivity disorder and observed classroom behavior. Journal of Consulting and Clinical Psychology, 73, 424-434.

Epstein, J. N., Casey, B. J., Tonev, S. T., Davidson, M. C., Reiss, A. L., Garrett, A., Hinshaw, S. P., Greenhill, L. L., Glover, G., Shafritz, K. M., Vitolo, A., Kotler, L. A., Jarrett, M. A., \& Spicer, J. (2007). ADHD and medication-related brain activation effects in concordantly affected parent-child dyads with ADHD. Journal of Child Psychology and Psychiatry, 48, 899-913.

Faraone, S. V., Biederman, J., \& Monuteaux, M. C. (2001). Attention deficit hyperactivity disorder with bipolar disorder in girls: Further evidence for a familial subtype? Journal of Affective Disorders, 64, 19-26.

Faraone, S. V., Biederman, J., Monuteaux, M. C., Doyle, A. E., \& Seidman, L. J. (2002). A psychometric measure of learning disability predicts educational failure four years later in boys with attention deficit hyperactivity disorder. Journal of Attention Disorders, 4, 220-230.

Fischer, M., Barkley, R. A., Smallish, L., \& Fletcher, K. (2005). Executive functioning in hyperactive children as young adults: Attention, inhibition, response perseveration, and the impact of comorbidity. Developmental Neuropsychology, 27, 107-133.

Frazier, T. W., Demaree, H. A., \& Youngstrom, E. A. (2004). Metaanalysis of intellectual and neuropsychological test performance in attention-deficit hyperactivity disorder. Neuropsychology, 18, 543-555.

Gadow, K. D., Drabick, D. A., Loney, J., Sprafkin, J., Salisbury, H., Azizian, A., \& Schwartz, J. (2004). Comparison of ADHD symptom subtypes as source-specific syndromes. Journal of Child Psychology and Psychiatry, 45, 1135-1149.

Gau, S. F. S. (2001). Individual and familial correlates and outcomes of attention deficit hyperactivity disorder: A longitudinal follow-up study of a high-risk sample (high-risk children). Dissertation Abstracts International: Section B - The Science \& Engenieering, 62, 1350.

Gershon, J. (2002). A meta-analytic review of gender differences in ADHD. Journal of Attention Disorders, 5, 143-154.

Geurts, H. M., Verté, S., Oosterlaan, J., Royers, H., \& Sergeant, J.A. (2004). How specific are executive function deficits in attention deficit hyperactivity disorder and autism? Journal of Child Psychology and Psychiatry, 45, 836-854. 
Geurts, H. M., Verté, S., Oosterlaan, J., Royers, H., \& Sergeant, J.A. (2005). ADHD subtypes: Do they differ in their executive functioning profile? Archives of Clinical Neuropsychology, 20, 457-477.

Gonzalez-Heydrich, J., Dodds, A., Whitney, J., MacMillan, C., Waber, D., Faraone, S. V., Boyer, K., Mrakotsky, C., DeMaso, D., Bourgeois, B., \& Biederman, J. (2007). Psychiatric disorders and behavioral characteristics of pediatric patients with both epilepsy and attention-deficit hyperactivity disorder. Epilepsy \& Behavior 10, 384-388.

Goos, L. M., Ezzatian, P., \& Schachar, R. (2007). Parent-of-origin effects in attention-deficit hyperactivity disorder. Psychiatry Research, 149, 1-9.

Graetz, B. W., Sawyer, M. G., Baghurst, P., \& Ettridge, K. (2006). Are ADHD gender patterns moderated by sample source? Journal of Attention Disorders, 10, 36-43.

Havey, J. M., Olson, J. M., McCormick, C., \& Cates, G. L. (2007). Teachers' perceptions of the incidence and management of attention-deficit hyperactivity disorder. Applied Neuropsychology, 12, 120-127.

Harpold, T., Biederman, J., Gignac, M., Hammerness, P., Surman, C., Potter, A., \& Mick, E. (2007). Is oppositional defiant disorder a meaningful diagnosis in adults?: Results from a large sample of adults with ADHD. Journal of Nervous and Mental Disease, 195, 601-605.

Herman, K. C., Lambert, S. F., Ialongo, N. S., \& Ostrander, R. (2007). Academic pathways between attention problems and depressive symptoms among urban African American children. Journal of Abnormal Child Psychology, 35, 265-274.

Hermens, D. F., Kohn, M. R., Clarke, S. D., Gordon, E., \& Williams, L. M. (2005). Sex differences in adolescent ADHD: Findings from concurrent EEG and EDA. Clinical Neurophysiology, 116, 1455-1463.

Hinshaw, S. P. (1994). Attention deficits and hiperactivity in children. Thousand Oaks: SAGE Publications.

Hinshaw, S. P., Carte, E. T., Sami, N., Treuting, J. J., \& Zupan, B. A. (2002). Preadolescent girls with attention-deficit hyperactivity disorder: Neuropsychological performance in relation to subtypes and individual classification. Journal of Consulting and Clinical Psychology, 70, 1099-1111.

Hinshaw, S. P., Carte, E. T., Fan, C., Jassy, J. S., \& Owens, E. B. (2007). Neuropsychological functioning of girls with attentiondeficit hyperactivity disorder followed prospectively into adolescence: Evidence for continuing deficits? Neuropsychology, 21, 263-273.

Huang-Pollock, C., Nigg, J. T., \& Halperin, J. M. (2006). Single dissociation findings of ADHD deficits in vigilance but not anterior or posterior attention systems. Neuropsychology, 20, 420-429.

Kadesjö, B., \& Gillberg, C. (2001). The comorbidity of ADHD in the general population of Swdish school-age children. Journal of Child Psychology and Psychiatry, 42, 487-492.

Kessler, R. C., Adler, L., Barkley, R., Biederman, J., Conners, C. K., Demler, O., Faraone, S. V., Greenhill, L. L., Howes, M. J., Secnik, K., Spencer, T., Ustun, T. B., Walters, E. E., \& Zaslavsky, A. M. (2006). The prevalence and correlates of adult ADHD in the United States: Results from the National Cormorbidity Survey Replication. American Journal of Psychiatry, 163, 716-23.
Kutcher, S., Aman, M., Brooks, S. J., Buitelaar, J., Van Daalen, E., Fegert, J., Findling, R. L., Fisman, S., Greenhill, L. L., Huss, M., Kusumakar, V., Pine, D., Taylor, E., \& Tyano, S. (2004). International consensus statement on attention-deficit/ hyperactivity disorder (ADHD) and disruptive behaviour disorders (DBDs): Clinical implications and treatment practice suggestions. European Neuropsychopharmacology, 14, 1128.

Lane, B. A. (2004). The differential neuropsychological/ cognitive profiles of ADHD subtypes: A meta-analysis. Dissertation Abstracts International: Section B - The Sciences \& Engineering, 64, 55-90.

Manga, D., Fournier, C., \& Navarredonda, A. B. (1999). Transtornos por déficit de atención en la infancia. Em A. Belloch, B. Sandín \& F. Ramos (Eds.), Manual de psicopatología, Vol. 2 (pp. 695 718). Madrid: McGraw-Hill.

Mannuzza, S., Klein, R. G., \& Moulton, J. L. (2002). Young adult outcome of children with "situational" hyperactivity: A prospective, controlled follow-up study. Journal of Abnormal Child Psychology, 30, 191-198.

Martinussen, R., \& Tannock, R. (2006). Working memory impairments in children with attention-deficit hyperactivity disorder with and without comorbid language learning disorders. Journal of Clinical and Experimental Neuropsychology, 28, 1073-1094.

MTA Cooperative Group (1999). Moderators and mediators of treatment response for children with attention-deficit hyperactivity disorder. Archives of General Psychiatry, 56, 1088-1096.

Murphy, K. R., Barkley, R. A., \& Bush, T. (2002). Young adults with attention deficit hyperactivity disorder: Subtype differences in comorbidity educational and clinical history. Journal of Nervous and Mental Disease, 190, 147-157.

Nadder, T. S., Rutter, M., Silberg, J. L., Maes, H. H., \& Eaves, L. J. (2002). Genetic effects on the variation and covariation of attention deficit-hyperactivity disorder (ADHD) and oppositional-defiant disorder/conduct disorder (ODD/ CD) symptomatologies across informant and occasion of measurement. Psychological Medicine, 32, 39-53.

Neuman, R. J., Sitdhiraksa, N., Reich, W., Ji, T. H-C., Joyner, C. A., Sun, L-W., \& Todd, R. D. (2005). Estimation of prevalence of DSM-IV and latent class-defined ADHD subtypes in a population-based sample of child and adolescent twins. Twin Research and Human Genetics, 8, 392-401.

Newcorn, J., Halperin, J. M., Jensen, P. S., Abikoff, H. B., Arnold, E., Cantwell, D. P., Conners, C. K., Elliott, G. R., Epstein, J. N., Greenhill, L. L., Hechtman, L., Hinshaw, S. P., Hoza, B., Kraemer, H. C., Pelham, W. E., Severe, J. B., Swanson, J. M., Wells, K. C., Wigal, T., \& Vitiello, B. (2001). Symptom profiles in children with ADHD: Effects of comorbidity and gender. Journal of the American Academy of Child \& Adolescent Psychiatry, 40, 137-146.

Nigg, J. T., Blaskey, L. G., Stawicki, J. A., \& Sachek, J. (2004). Evaluating the Endophenotype Model of ADHD neuropsychological deficit: Results for parents and siblings of children with ADHD combined and inattentive subtypes. Journal of Abnormal Psychology, 113, 614-625. 
NIH (2000). National Institutes of Health Consensus Development Conference Statement: Diagnosis and treatment of attentiondeficit/hyperactivity disorder (ADHD). Journal of the American Academy of Child and Adolescent Psychiatry, 39, 182-193.

Nøvik, T. S., Hervas, A., Ralston, S. J., Dalsgaard, S., Pereira, R. R., \& Lorenzo, M. J. (2006). Influence of gender on attentiondeficit hyperactivity disorder in Europe - ADORE. European Child \& Adolescent Psychiatry, 15 (1, Suppl.), 15-24.

Ostrander, R., Crystal, D. S., \& August, G. (2006). Attentiondeficit hyperactivity disorder, depression, and self- and otherassessments of social competence: A developmental study. Journal of Abnormal Child Psychology, 34, 773-787.

Pasini, A., Paloscia, C., Alessadrelli, R., Porfirio, M. C., \& Curatolo, P. (2007). Attention and executive functions profile in drug naïve ADHD subtypes. Brain \& Development, 29, 400-408.

Polanczyk G, de Lima, M. S., Horta, B. L., Biederman, J., \& Rohde, L. A. (2007). The worldwide prevalence of ADHD: A systematic review and metaregression analysis. The American Journal of Psychiatry, 164, 942-948.

Power, T. J., Costigan, T. E., Eiraldi, R. B., \& Leff, S. S. (2004). Variations in anxiety and depression as a function of ADHD subtypes defined by $D S M-I V$ : Do subtype differences exist or not? Journal of Abnormal Child Psychology, 3, 27-37.

Raymaekers, R., Antrop, I., Van der Meere, J. J., Wiersema, J. R., \& Roeyers, H. (2007). HFA and ADHD: A direct comparison on state regulation and response inhibition. Journal of Clinical and Experimental Neuropsychology, 29, 418-427.

Robertson, M. M. (2006). Attention deficit hyperactivity disorder, tics and Tourette's syndrome: The relationship and treatment implications. A commentary. European Child \& Adolescent Psychiatry, 15, 1-11.

Rucklidge, J. J., \& Tannock, R. (2002). Neuropsychological profiles of adolescents with ADHD: Effects of reading difficulties and gender. Journal of Child Psychology and Psychiatry and Allied Disciplines, 43, 988-1003.

Rumsey, R. K. (2004). Executive functioning in boys and girls with attention-deficit hyperactivity disorder with and without a comorbid reading disability. Dissertation Abstracts International: Section B - The Sciences \& Engineering, 64, 5820 .

Schatz, D. B., \& Rostain, A. L. (2006). ADHD with comorbid anxiety: A review of the current literature. Journal of Attention Disorders, 10, 141-149.

Scheres, A., Oosterlaan, J., Geurts, H., Morein-Zamir, S., Meiran, N., Schut, H., Vlasveld, L., \& Sergeant, J.A. (2004). Executive functioning in boys with ADHD: Primarily an inhibition deficit? Archives of Clinical Neuropsychology, 19, 569-594.

Seidman, L. J., Biederman, J., Monuteaux, M. C., Valera, E., Doyle, A. E., \& Faraone, S. V. (2005). Impact of gender and age on executive functioning: Do girls and boys with and without attention deficit hyperactivity disorder differ neuropsychologically in preteen and teenage years? Developmental Neuropsychology, 27, 79-105.

Seidman, L. J. (2006). Neuropsychological functioning in people with ADHD across the lifespan. Clinical Psychology Review, $26,466-485$.

Sergeant, J. A., Geurts, H. M., \& Oosterlaan, J. (2002). How specific is a deficit of executive functioning for AttentionDeficit/Hyperactivity Disorder? Behavioural Brain Research, 130, 3-28.
Short, E. J., Fairchild, L., Findling, R. L., \& Manos, M. J. (2007). Developmental and subtype differences in behavioral assets and problems in children diagnosed with ADHD. Journal of Attention Disorders, 11, 28-36.

Skounti, M., Philalithis, A., \& Galanakis, E. (2007). Variations in prevalence of attention deficit hyperactivity disorder worldwide. European Journal of Pediatrics, 166, 117-23.

Souza, I., Serra, M. A., Mattos, P., \& Franco, V. A. (2001). Comorbidade em crianças e adolescentes com transtorno do deficit de atenção: Resultados preliminares. Arquivos de Neuropsiquiatria, 59, 401-406.

Spencer, T. J., Biederman, J., \& Mick, E. (2007). Attention-deficit hyperactivity disorder: Diagnosis, lifespan, comorbidities, and neurobiology. Ambulatory Pediatrics, 7, 73-81.

Staller, J., \& Faraone, S. V. (2006). Attention-deficit hyperactivity disorder in girls epidemiology and management. CNS Drugs, 20, 107-123.

Taylor, E., Dopfner, M., Sergeant, J., Asherson, P., Banaschewski, T., Buitelaar, J., Coghill, D., Danckaerts, M., Rothenberger, A., Sonuga-Barke, E., Steinhausen, H. C., \& Zuddas, A. (2004). European clinical guidelines for hyperkinetic disorder - first upgrade. European Child \& Adolescent Psychiatry, 13 (1, Suppl.), 17-130.

Thapar, A., Harrington, R., McGuffin, P. (2001) Examining the comorbidity of ADHD related behaviours and conduct problems using a twin study design. British Journal of Psychiatry, 179, 224-229.

Thapar, A., Langley, K., O’Donovan, M., \& Owen, M. (2006). Refining the attention deficit hyperactivity disorder phenotype for molecular genetic studies. Molecular Psychiatry, 11, 714720.

Warner-Rogers, J., Taylor, A., Taylor, E., \& Sandberg, S. (2000). Inattentive behavior in childhood. Journal of Learning Disabilities, 33, 520-537.

Willcutt, E. G., Pennington, B. F., Olson, R. K., Chabildas, N., \& Hulslander, J. (2005). Neuropsychological analyses of comorbidity between reading disability and attention deficit hyperactivity disorder: In search of the common deficit. Developmental Neuropsychology, 27, 35-78.

Willcutt E. G., Pennington, B. F., Olson, R. K., \& DeFries, J. C. (2007). Understanding comorbidity: A twin study of reading disability and attention-deficit hyperactivity disorder. American Journal of Medical Genetics, 144B, 709-714.

World Health Organization (1996). Multiaxial classification of child and adolescent psychiatric disorders. The ICD-10 classification of mental and behavioral disorders in children and adolescents. Cambridge: Cambridge University Press.

Recebido em 18.05 .08

Primeira decisão editorial em 14.08.08

Versão final em 04.09.08

Aceito em 20.11.08 